\title{
Leisure time physical activity is associated with improved HDL functionality in high cardiovascular risk individuals: a cohort study
}

European Journal of Preventive Cardiology $0(0) \mathrm{I}-12$

(C) The European Society of Cardiology 2020 Article reuse guidelines: sagepub.com/journals-permissions DOI: $10.1177 / 2047487320925625$ journals.sagepub.com/home/cpr @SAGE

\author{
Álvaro Hernáez 1,2,3, Maria Trinidad Soria-Florido', \\ Olga Castañer ${ }^{1,3}$, Xavier Pintó ${ }^{3,4}$, Ramón Estruch ${ }^{2,3,5}$, \\ Jordi Salas-Salvadó ${ }^{3,6}$, Dolores Corella ${ }^{3,7}$, \\ Ángel Alonso-Gómez ${ }^{3,8}$, Miguel Ángel Martínez-González 3,9,10, \\ Helmut Schröder ${ }^{1,1}$, Emilio Ros ${ }^{3,5}$, Lluis Serra-Majem ${ }^{3,12}$, \\ Miquel Fiol $^{3,13}$, José Lapetra ${ }^{3,14}$, Enrique Gomez-Gracia ${ }^{3,15}$, \\ Montserrat Fitó ${ }^{1,3}$ and Camille Lassale ${ }^{1,3,16}$
}

\begin{abstract} with poorer high-density lipoprotein vasodilatory capacity.

\footnotetext{
'Hospital del Mar Medical Research Institute (IMIM), Spain

${ }^{2}$ August $\mathrm{Pi}$ i Sunyer Biomedical Research Institute (IDIBAPS), Spain

${ }^{3}$ CIBER of Pathophysiology of Obesity and Nutrition (CIBEROBN), Instituto de Salud Carlos III, Spain

${ }^{4}$ Hospital Universitario de Bellvitge, L'Hospitalet de Llobregat, Spain

${ }^{5}$ Hospital Clínic Barcelona, Spain

${ }^{6}$ Hospital Universitari Sant Joan de Reus, Universitat Rovira i Virgili, Spain

${ }^{7}$ Universidad de Valencia, Spain

${ }^{8}$ Bioaraba Health Research Institute, University of the Basque Country UPV/EHU, Spain

'Universidad de Navarra, Spain

${ }^{10}$ Harvard TH Chan School of Public Health, USA
}

Aims: Physical activity has consistently been shown to improve cardiovascular health and high-density lipoproteincholesterol levels. However, only small and heterogeneous studies have investigated the effect of exercise on highdensity lipoprotein functions. Our aim is to evaluate, in the largest observational study to date, the association between leisure time physical activity and a range of high-density lipoprotein functional traits.

Methods: The study sample consisted of 296 Spanish adults at high cardiovascular risk. Usual leisure time physical activity and eight measures of high-density lipoprotein functionality were averaged over two measurements, one year apart. Multivariable linear regression models were used to explore the association between leisure time physical activity (exposure) and each high-density lipoprotein functional trait (outcome), adjusted for cardiovascular risk factors.

Results: Higher levels of leisure time physical activity were positively and linearly associated with average levels over one year of plasma high-density lipoprotein-cholesterol and apolipoprotein A-I, paraoxonase-I antioxidant activity, highdensity lipoprotein capacity to esterify cholesterol and cholesterol efflux capacity in individuals free of type 2 diabetes only. The increased cholesterol esterification index with increasing leisure time physical activity reached a plateau at around $\mathbf{3 0 0}$ metabolic equivalents.min/day. In individuals with diabetes, the relationship with cholesteryl ester transfer protein followed a U-shape, with a decreased cholesteryl ester transfer protein activity from 0 to 300 metabolic equivalents.min/day, but increasing from there onwards. Increasing levels of leisure time physical activity were associated

Conclusions: In a high cardiovascular risk population, leisure time physical activity was associated not only with greater circulating levels of high-density lipoprotein-cholesterol, but also with better markers of high-density lipoprotein functionality, namely cholesterol efflux capacity, the capacity of high-density lipoprotein to esterify cholesterol and

I'CIBER Epidemiology and Public Health (CIBERESP), Instituto de Salud Carlos III, Spain

${ }^{12}$ Universidad de Las Palmas de Gran Canaria, Spain

${ }^{13}$ Balearic Islands Health Research Institute, Hospital Son Espases, Spain

${ }^{14}$ Distrito Sanitario Atención Primaria Sevilla, Spain

${ }^{15}$ Universidad de Málaga, Spain

${ }^{16}$ Department of Epidemiology and Public Health, University College London, UK

\section{Corresponding author:}

Camille Lassale, Cardiovascular Risk and Nutrition Research Group, Program of Epidemiology and Public Health, Hospital del Mar Medical Research Institute (IMIM) PRBB, Carrer Doctor Aiguader 88, 08003 Barcelona, Spain.

Email: classale@imim.es 
paraoxonase-I antioxidant activity in individuals free of diabetes and lower cholesteryl ester transfer protein activity in individuals with type 2 diabetes.

\section{Keywords}

HDL function, physical activity, lifestyle, biomarkers

Received 6 November 2019; accepted 22 April 2020

\section{Introduction}

Diet and physical activity are key lifestyle factors that can modulate the risk of developing atherosclerotic disease. ${ }^{1}$ Epidemiological studies have consistently found that low levels of high-density lipoprotein (HDL) cholesterol are associated with atherosclerotic cardiovascular disease. ${ }^{2}$ Nevertheless, the causal role of HDL-cholesterol on cardiovascular disease risk has been challenged by Mendelian randomisation studies $^{3-5}$ and unsuccessful pharmacological interventions. ${ }^{6,7}$ This has led to the hypothesis that improving HDL function can be more relevant for cardiovascular prevention than raising HDL-cholesterol concentrations. ${ }^{8}$ Although it is established that exercise increases HDL-cholesterol levels, ${ }^{9}$ its effects on HDL functionality have been less studied and remain to be clarified. As reviewed recently, there is emerging evidence that exercise interventions can improve aspects of HDL functionality in populations at elevated cardiovascular risk (sedentary, overweight/obese and/or with metabolic syndrome in the majority of studies). ${ }^{10}$ The highest level of evidence was found for cholesterol efflux capacity (CEC), with results from rigorously controlled, large exercise training interventions showing that regular prolonged vigorous exercise improves CEC. For example, in a study of adults with metabolic syndrome weight loss and exercise during 3 months was associated with a $25 \%$ increase in CEC. ${ }^{11}$ Another study compared different exercise intensities during a 6-month intervention and concluded that CEC only improved with a high dose of high intensity training. ${ }^{12}$ However, only a few studies with small samples (median $n=33$, median duration 3 months) of individuals with metabolic disorders have reported beneficial effects of exercise intervention on other HDL functional properties such as anti-inflammatory and antioxidant. ${ }^{10,13}$ Moreover, no observational study has described the effects of real-life levels of physical activity in at-risk populations on a comprehensive set of HDL function traits.

Therefore, the aim of this study was to assess comprehensively the relationship between physical activity and a range of markers of HDL characteristics and functionality in a sample of nearly 300 individuals at high risk of cardiovascular disease.

\section{Methods}

\section{Study population}

The PREDIMED trial (PREvencion con DIeta MEDiterranea) is a dietary intervention which consisted of following a traditional Mediterranean diet supplemented with either virgin olive oil or nuts, and the control group followed a low-fat diet. ${ }^{14}$ Individuals were free of cardiovascular disease but had either type 2 diabetes or at least three of the following cardiovascular risk factors: current smoking, hypertension, increased low-density lipoprotein (LDL) cholesterol, decreased HDL-cholesterol, overweight/obesity or a family history of premature heart disease. The local research and ethics committee approved the study protocol. All participants gave written informed consent. The trial is registered at http://www.controlled-trials. com/ISRCTN35739639.

The population used for this analysis was a random subsample of 296 participants from the PREDIMED trial, ${ }^{14,15}$ with equal proportions of participants in each arm of the study (control, Mediterranean diet plus extra virgin olive oil, Mediterranean diet plus nuts) in which an extensive assessment of HDL functions has been performed at baseline and at one year of followup. ${ }^{15}$ The sample size of 296 was calculated to detect a significant difference of 0.025 points in normalised CEC values between pre and post-intervention values in the seminal paper that investigated the effect of a Mediterranean dietary intervention on HDL functionality. ${ }^{16}$ Only six out of the 11 recruiting centres of the PREDIMED trial could provide blood samples for the determination of HDL functionality. Frozen blood samples were shipped to Hospital del Mar Research Institute (IMIM), Barcelona, where all the HDL function assays were performed.

\section{Outcome assessment: HDL function}

A detailed description of HDL function assays, including coefficients of variation and detailed protocols, has 
been published elsewhere. ${ }^{16,17}$ Participants' HDLcholesterol and apolipoprotein A-I (ApoA-I) levels were analysed in an ABX-Pentra 400 autoanalyzer (Horiba ABX, Montpellier, France). HDL particles were first isolated from plasma samples by density gradient ultracentrifugation (isolated HDL fraction) or polyethylene glycol-induced precipitation of apolipoprotein B-containing lipoproteins (apolipoprotein B depleted plasma), and the samples were stored at $-80^{\circ} \mathrm{C}$ until use. The following properties were determined as previously described: ${ }^{16,17}$ (a) CEC in a model of human THP-1 monocyte-derived macrophages with $3 \mathrm{H}$-cholesterol treated with $5 \%$ apolipoprotein B-depleted plasma samples; (b) the ability of HDLs to esterify cholesterol (HDL esterification index) as the ratio between the percentage of esterified cholesterol in isolated HDL plasma samples and lecithin cholesterol acyltransferase concentration in serum samples; (c) HDL inflammatory index, an indirect measurement of the ability of apolipoprotein B-depleted particles to protect LDL from oxidation, based on the oxidation of the fluorescent $2^{\prime}, 7^{\prime}$-dichlorodihydrofluorescein; (d) the activities of cholesteryl ester transfer protein (CETP) and paraoxonase-1 (PON1) enzymes in plasma and serum samples, respectively, by commercial kits; (e) HDL vasodilatory capacity as the capacity to promote endothelial release of nitric oxide in vitro in a human umbilical vein endothelial cell model treated with apolipoprotein B-depleted plasma samples. Due to sample availability and technical issues, there were 67 missing values for CETP and PON1 activity, and 50 missing for HDL vasodilatory capacity. The cumulative average of the baseline and one-year follow-up measure was calculated and used as primary outcome variable, to increase precision. ${ }^{18}$

\section{Exposure assessment: physical activity}

Non-occupational physical activity was assessed at baseline and at one year by the self-administered Minnesota leisure time physical activity (LTPA) questionnaire. The Spanish version, consisting of 67 activities organised in nine sections, has been validated in men and women. ${ }^{19,20}$ Participants were asked to indicate the number of days and minutes per day they had performed those activities during the previous year. Physical activity was quantified in metabolic equivalents (METs) of task in minutes per day by multiplying the METs of each activity with its mean duration (in minutes per day). LTPA was classified as light LTPA (intensity $<4$ METs, e.g. walking), moderate LTPA (4-5.5 METs, e.g. brisk walking) and vigorous ( $\geq 6$ METs, e.g. jogging). Similar to the outcomes, the cumulative average of the baseline and one-year follow-up measure was calculated and used as a primary exposure variable, to reflect usual physical activity better.

\section{Covariates}

Clinical variables were measured by a trained nurse (weight, height, blood pressure and biochemical profile). Questionnaires were used to collect information on medication, smoking habits and adherence to a traditional Mediterranean diet by a validated 14-item screener. ${ }^{21}$ Body mass index (BMI) was calculated as the ratio between weight $(\mathrm{kg})$ and the height squared $\left(\mathrm{m}^{2}\right)$. BMI and Mediterranean diet score were averaged over the baseline and one-year values. Dyslipidemia was defined by the presence of any of the following: hypercholesterolemia defined as total cholesterol $200 \mathrm{mg} \mathrm{dL}^{-1}$ or greater or the use of statins; hypertriglyceridemia defined as triglycerides $150 \mathrm{mg} \mathrm{dL}^{-1}$ or greater and/or the use of fibrates or pharmacological doses of omega-3 polyunsaturated fatty acids. Type 2 diabetes mellitus was defined as the presence of an altered glucose metabolism or the use of antidiabetic drugs. Hypertension was defined as systolic blood pressure $140 \mathrm{mmHg}$ or greater, diastolic blood pressure 90 $\mathrm{mmHg}$ or greater, or the use of antihypertensive agents.

\section{Statistical analysis}

Quintiles of the cumulative average of physical activity were created. We describe the sample characteristics overall across quintiles of LTPA and calculated the $P$ value for linear trend across quintiles using contrasts. For the main analysis, the outcomes of interest were each HDL function trait (cumulative average), standardised to z-scores with a mean of 0 and a standard deviation of 1 , so that all estimates can be comparable. We used separate generalised linear models for each HDL function trait as dependent variables, and different levels of LTPA as independent variables, treated as continuous. The beta coefficients from these regressions are given for an increase in 100 METs.min/day. This corresponds, for example, to 25 minutes of brisk walking (a moderate physical activity, estimated at 4 METs) per day, thus 175 minutes per week. The main results are given for LTPA as the sum of light, moderate and vigorous LTPA. Diabetes being a strong correlate of HDL functionality, ${ }^{22}$ we tested for interaction by introducing a cross-product term between LTPA and diabetes status, and stratified the analyses by type 2 diabetes status. To investigate the shape of the association, LTPA variables were also modelled in quintiles and predicted adjusted means across quintiles are presented. All models were adjusted for sex, intervention group, study centre and baseline age, BMI, 
smoking status, score of adherence to a traditional Mediterranean diet, dyslipidemia, type 2 diabetes and hypertension status. To assess better the potential nonlinear dose-response relationship, restricted cubic splines were fitted. Likelihood ratio tests between fully adjusted nested models, the first with only the linear term and the second with the restricted cubic spline terms were performed. The resulting $P$ value is denoted as ' $P$ value non-linear'. To avoid distortion of the spline curves by extreme values, we excluded LTPA values greater than 1000 METs.min/day $(n=4)$ for the spline analysis. All analyses were conducted using Stata 14.0 (Stata Corp) and the 'rcs' package in R Software, version 3.5.0. All tests were two-sided with an alpha level of 0.05 .

\section{Results}

Participants' characteristics are described in Table 1. Median LTPA was 201 METs.min/day, coming mostly from activities of low intensity (115 METs. $\mathrm{min}$ (day). Expressed in METs.min/week, this is 1407 METs.min/week, which is more than twice the minimum recommended by the World Health Organization of 600 METs.min/week of total LTPA. The levels of LTPA in the first quintile (2.0-86.5 METs.min/day) are below the recommended minimum amount. The majority of characteristics did not show a statistically significant trend across quintiles of LTPA, with the exception of a lower proportion of women $P<0.001$.

As seen in Figure 1 by quintile and Figure 2 in the spline analysis, higher levels of LTPA were positively and linearly associated with HDL-cholesterol concentrations after adjustment for confounders, despite not reaching conventional statistical significance $\left(\beta_{100 \mathrm{METs}}=0.058 \quad(95 \% \quad\right.$ confidence interval $(\mathrm{CI})$ $-0.007,0.123), P=0.081)$. Unsurprisingly, as it is the major protein constituent of HDL-cholesterol, plasma ApoA-I levels also tended to increase as LTPA levels did, although reaching a plateau after 400 METs.min/ day, which corresponds to the fifth quintile. Regarding HDL functionality, there was evidence of a positive association of LTPA with PON1 antioxidant activity $\left(\beta_{100 \mathrm{METs}}=0.070(95 \% \mathrm{CI}-0.008,0.148), P=0.079\right)$. The relationship with HDL capacity to esterify cholesterol was not linear $(P$ value non-linear $=0.078)$, and a model using restricted cubic splines with three knots shows that the increase observed in the cholesterol esterification index from 0 to $300 \mathrm{METs}$.min/day plateaus from 300 METs.min/day onwards (Figure 2). The relationship with CETP was also non-linear, as the model with cubic splines had a significantly better fit $(P=0.022)$, and reveals a U-shape, with decreasing CETP activity as LTPA increased from 0 to
300 METs.min/day, where it then plateaued until 400 METs.min/day, beyond which CETP activity increased as LTPA increased (Figure 2). Surprisingly, increasing levels of LTPA were associated with lower HDL vasodilatory capacity (Figure $1, \beta_{100 \mathrm{METs}}=-0.058(95 \% \mathrm{CI}$ $-0.115,-0.001), P=0.049)$. However, the cubic spline analysis revealed that a model with seven knots had a significantly better fit than the linear model $(P=0.020)$, showing an oscillating relationship (Figure 2). There was no clear evidence of any association between LTPA and CEC, nor with the HDL inflammatory index. The splines show that, for the HDL functional traits that display an association with physical activity, compared with no physical activity (0 METs.min/day), the effects were significant from approximately 80-100 METs.min/day (where the confidence interval crosses the $\mathrm{x}$-axis), which corresponds to the minimum amount of physical activity recommended by the World Health Organization. ${ }^{23}$

The characteristics of participants according to diabetes status are presented in Supplementary Table 1. When stratifying by diabetes status (Figures 3 and 4), all associations, except for CETP, were only apparent in individuals without diabetes. In this group, we observed that LTPA was strongly and linearly associated with HDL-cholesterol, apolipoprotein A-I, PON1 antioxidant activity as well as CEC, and with HDL capacity to esterify cholesterol with a plateau at 300 METs.min/day. The associations with these traits were non-existent in individuals with diabetes. Conversely, LTPA displayed a U-shaped association with CETP activity in the diabetes group, but not in individuals without diabetes. The inverse association with vasodilatory index was also more apparent in people with diabetes than in those without diabetes.

\section{Discussion}

In a sample of 296 individuals at high cardiovascular risk, we found that LTPA was associated not only with greater circulating levels of HDL-cholesterol and ApoA-I, but also with better markers of HDL functionality, namely greater CEC, capacity of HDL to esterify cholesterol and PON1 antioxidant activity in subjects without diabetes, and lower CETP activity in individuals with diabetes.

Physical activity has been shown to increase HDLcholesterol levels in a large number of human trials and observational studies, ${ }^{8}$ even in subjects at elevated risk of cardiovascular disease. ${ }^{24}$ Our results support this association, which has been traditionally explained by the capacity of aerobic exercise to promote the activation of adenosine monophosphate-activated protein kinase (AMPK). ${ }^{25}$ AMPK is a key element involved in the regulation of cell metabolism at states of low 


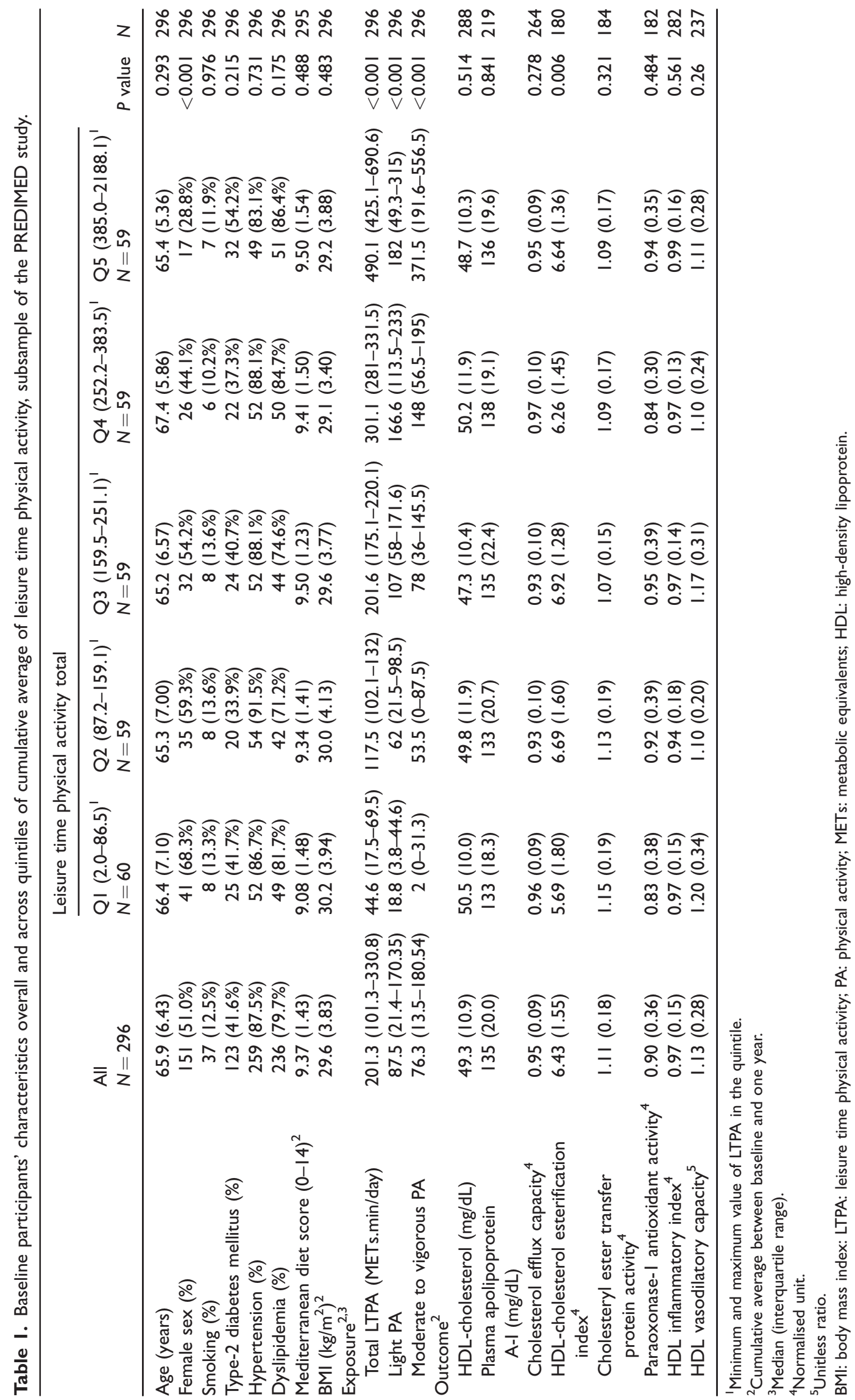




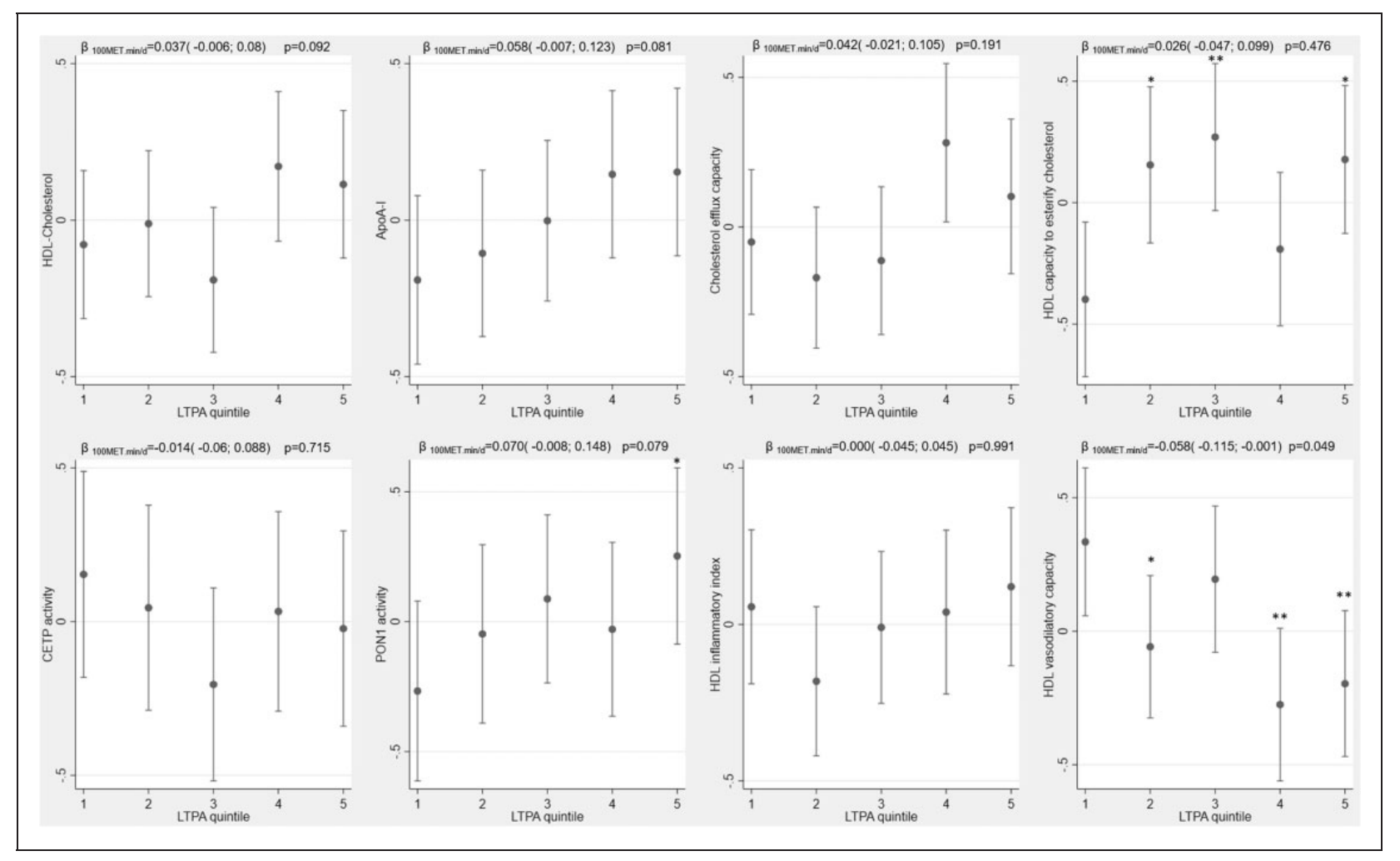

Figure I. Multivariable $\nmid$ estimated standardised mean z-score values and $95 \%$ confidence intervals of high-density lipoprotein (HDL)related traits across quintiles of leisure time physical activity (LTPA) and multivariable regression coefficients from generalized linear model regressions.

†Estimates are adjusted mean z-score and 95\% confidence intervals in each quintile of LTPA, adjusted for sex, intervention group, study centre and baseline age, body mass index, smoking status, Mediterranean diet score, dyslipidemia, type 2 diabetes and hypertension status. Significance of the comparison with the first quintile is given by the asterisks on the graph $* P<0.05$, $* * P<0.01$.

metabolic energy. ${ }^{26}$ Its activation seems to be partially responsible for the benefits of physical activity on lipid metabolism, glucose homeostasis, antioxidant/antiinflammatory protection and other pathways involved in the development of chronic diseases. ${ }^{27}$ Regarding HDL-related specific actions, AMPK is able to stimulate the peroxisome proliferator-activated receptor alpha, a transcription factor capable of promoting the hepatic synthesis of ApoA-I, leading to increases in HDL-cholesterol circulating levels. ${ }^{28}$ Moreover, its stimulation could also be partially responsible for the promotion of HDL anti-atherosclerotic functions.

Beyond the increments in the quantity of cholesterol circulating in HDL particles, we observed in the present study an association between LTPA and greater values of CEC, HDL ability to esterify cholesterol, and the activity of the HDL-bound antioxidant enzyme PON1. Despite not directly comparable due to design differences, the observed results of CEC are in line with outcomes of exercise interventions, lasting between 3 and 12 months in cardiovascular disease patients or individuals at elevated cardiovascular disease risk, that showed overall improvements in CEC up to a
$25 \%$ increase. $^{10}$ Shorter interventions (median of 3 months) also indicate some antioxidant effect of exercise on the ability of HDL to transport lipid peroxides. $^{10,13}$ Antioxidant properties of AMPK make biologically plausible the association of LTPA with these traits. On the one hand, AMPK is known to be able to promote the phosphorylation of forkhead box protein $\mathrm{O} 1$, a transcription factor capable of upregulating the expression of antioxidant enzymes such as superoxide dismutase and catalase. ${ }^{29,30}$ On the other hand, AMPK is also able to induce the activation of the nuclear factor erythroid 2-related factor 2, another transcriptional regulator capable of promoting the expression of antioxidant enzymes such as $\mathrm{NAD}(\mathrm{P}) \mathrm{H}$ dehydrogenase or glutathione S-transferase, among others. ${ }^{31}$ The combination of the previous two mechanisms may result in fewer oxidative modifications of HDL proteins, such as ApoA-I, lecithin cholesterol acyltransferase and PON1. These three proteins play a pivotal role in CEC, HDL capacity to esterify cholesterol and antioxidant defences, and are known partially to lose their function when they become oxidised. ${ }^{32-34}$ Therefore, an AMPK-mediated antioxidant 


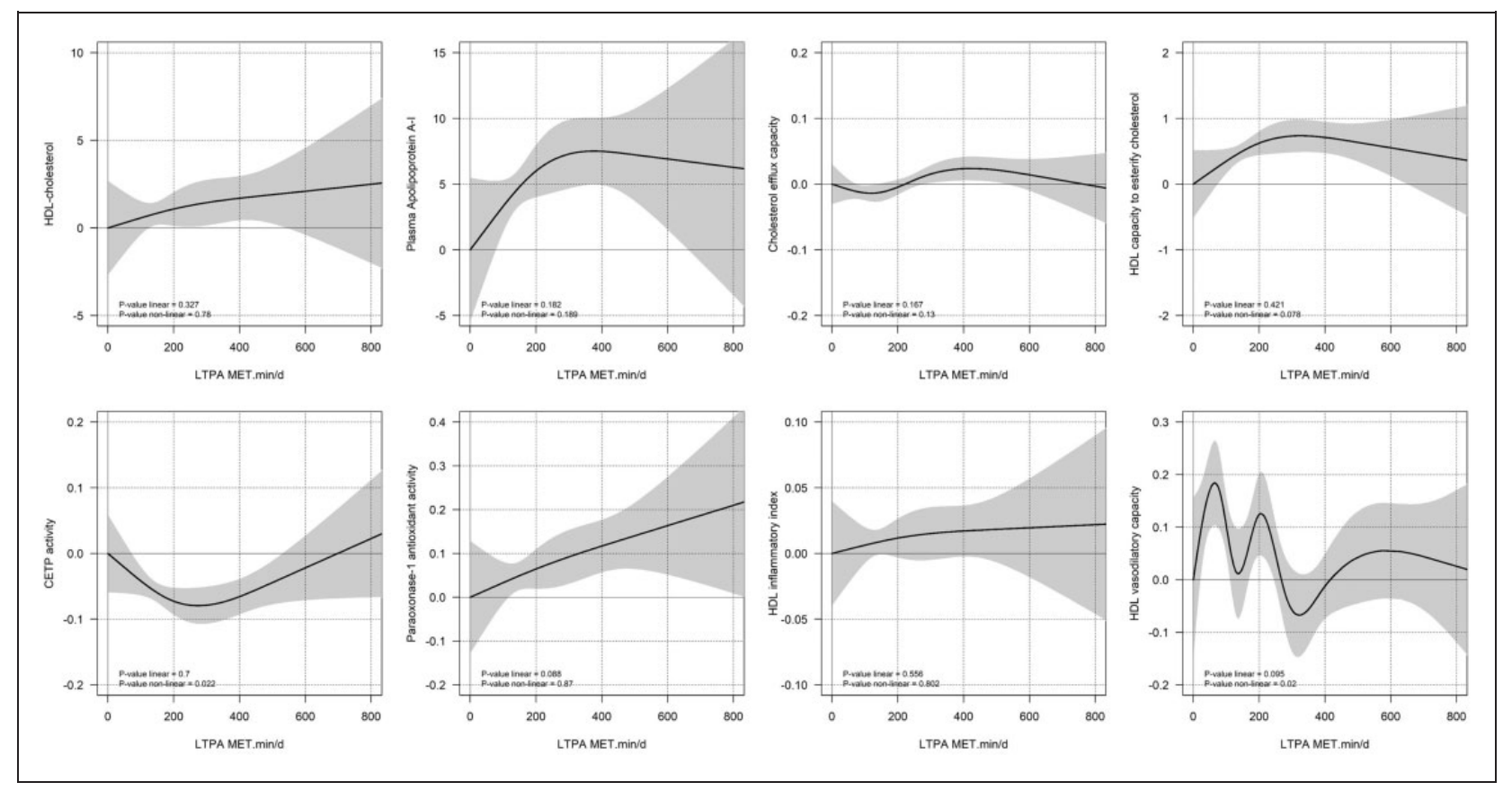

Figure 2. Dose-response association $\dagger$ of high-density lipoprotein (HDL)-related functional traits assessed by restricted cubic splines.

†Adjusted for sex, intervention group, study centre and baseline age, body mass index, smoking status, Mediterranean diet score, dyslipidemia, type 2 diabetes and hypertension status.

protection could contribute to explaining the improved HDL functions in individuals with greater levels of LTPA. There was no clear association with the HDL inflammatory index. Intervention studies are scant and have failed to produce consistent results on the effect of exercise on HDL anti-inflammatory capacity. ${ }^{10,13}$ A recent study in patients with hypertension found an effect of an aerobic intervention on the antioxidant but not anti-inflammatory capacity of HDL, measured by cell-free assays. ${ }^{13}$

HDL is more dysfunctional in individuals with type 2 diabetes, ${ }^{22}$ therefore to rule out the confounding effect of diabetes, we stratified our results and found that most associations were only apparent in people without diabetes. The interpretation of these results is that there is more scope for an improvement of HDL function by increasing physical activity levels in patients without diabetes, whereas in type 2 diabetes patients, the functionality of HDL shows little difference according to physical activity. This is in line with a recent review of the evidence that found only a limited effect of exercise training, in particular of resistance training, on lipid parameters in type 2 diabetes patients. ${ }^{35}$ The exception was for CETP activity, that displayed a U-shaped relationship with LTPA only in people with diabetes. We observed a decrease in the CETP activity with increasing physical activity up to moderate/high levels (300 METs-min/day), which correspond to approximately one hour of brisk walking or 30 minutes of jogging per day, every day. Considering that this enzyme requires a source of triglycerides to exchange for cholesteryl esters from HDL particles, ${ }^{36}$ and that circulating triglyceride levels are greater in diabetes patients, a decrease in triglycerides in the circulation, related to the physical activity practice, may be partially responsible for a slight moderation in its function. ${ }^{24}$

Finally, we observed that HDL vasodilatory capacity, measured by the release of nitric oxide, seemed to decrease when levels of LTPA increase, although the relationship was unclear and showed oscillations in the spline analysis that are not interpretable. The majority of studies that have studied the effect of exercise on nitric oxide have investigated its immediate acute effect on nitric oxide release in exhaled air. Those studies show either increased, decreased or unchanged levels of exhaled nitric oxide in response to exercise, probably depending on factors such as the levels of nitric oxide synthase, the severity of oxidative stress, nitric oxide binding to antioxidant molecules, and also individual patterns of physical activity. ${ }^{37}$ Moreover, long-term exposure to physical activity is not comparable with short-term changes, and the association observed here may also be mediated by potential changes in some key HDL components, such as acute phase HDL proteins. Due to the ambivalent 


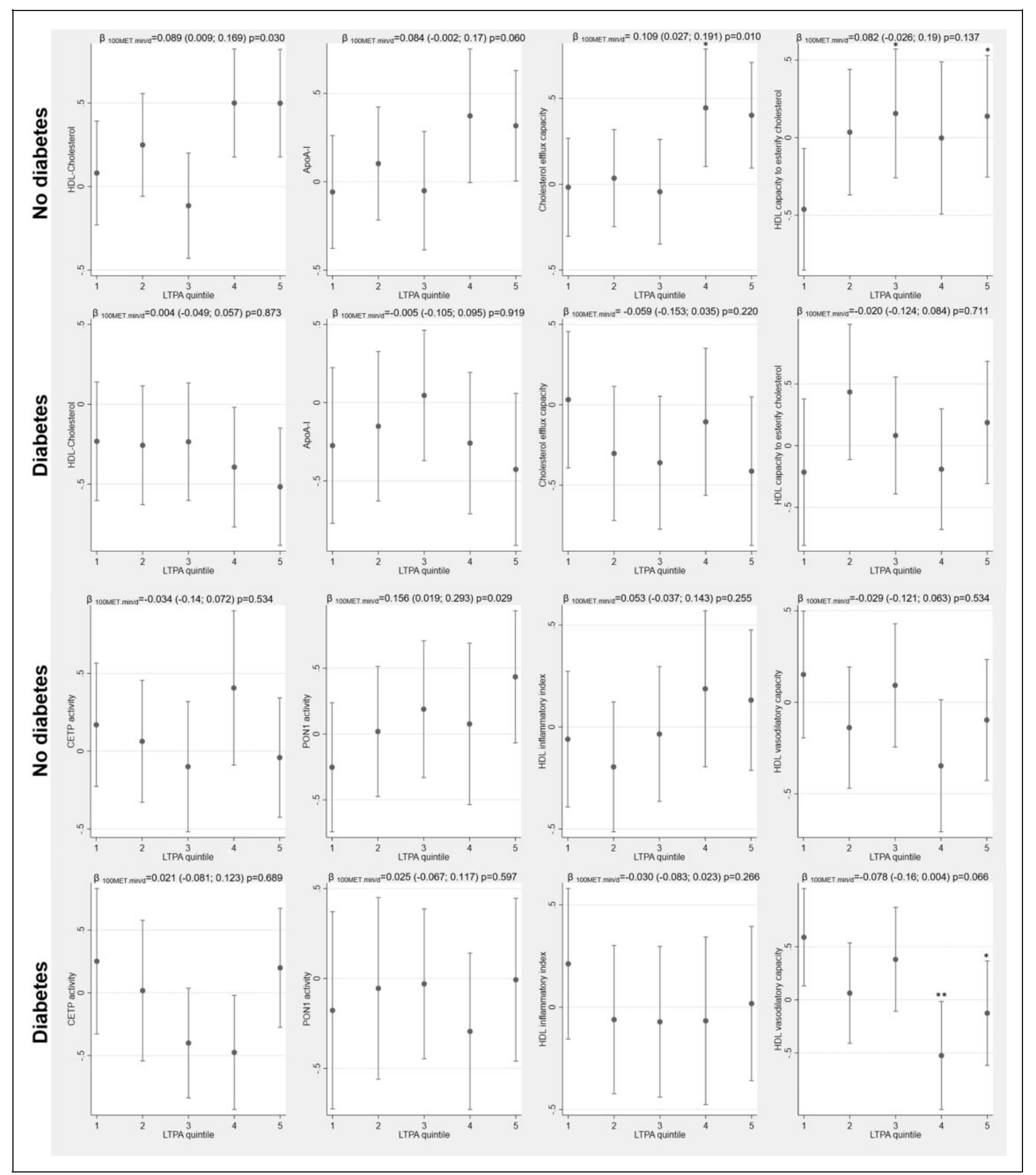

Figure 3. Multivariable $\nmid$ estimated standardised values and $95 \%$ confidence intervals of high-density lipoprotein (HDL)-related traits across quintiles of leisure time physical activity and multivariable regression coefficients from generalised linear model regressions by diabetes status.

†Estimates are adjusted for sex, intervention group, study centre and baseline age, body mass index, smoking status, Mediterranean diet score, dyslipidemia and hypertension status. Significance of the comparison with the first quintile is given by the asterisks on the graph $* P<0.05$, ** $P<0.0$ I.

$P$ value for interaction between leisure time physical activity (LTPA) and diabetes: HDL-cholesterol $P=0.305$, apolipoprotein A-I $P=0.673$, cholesterol efflux capacity $P=0.242$, HDL-cholesterol esterification index $P=0.100$, cholesteryl ester transfer protein activity $P=0.078$, paraoxonase- I antioxidant activity $P=0.054$, HDL inflammatory index $P=0.152$, HDL vasodilatory capacity $P=0.719$. 


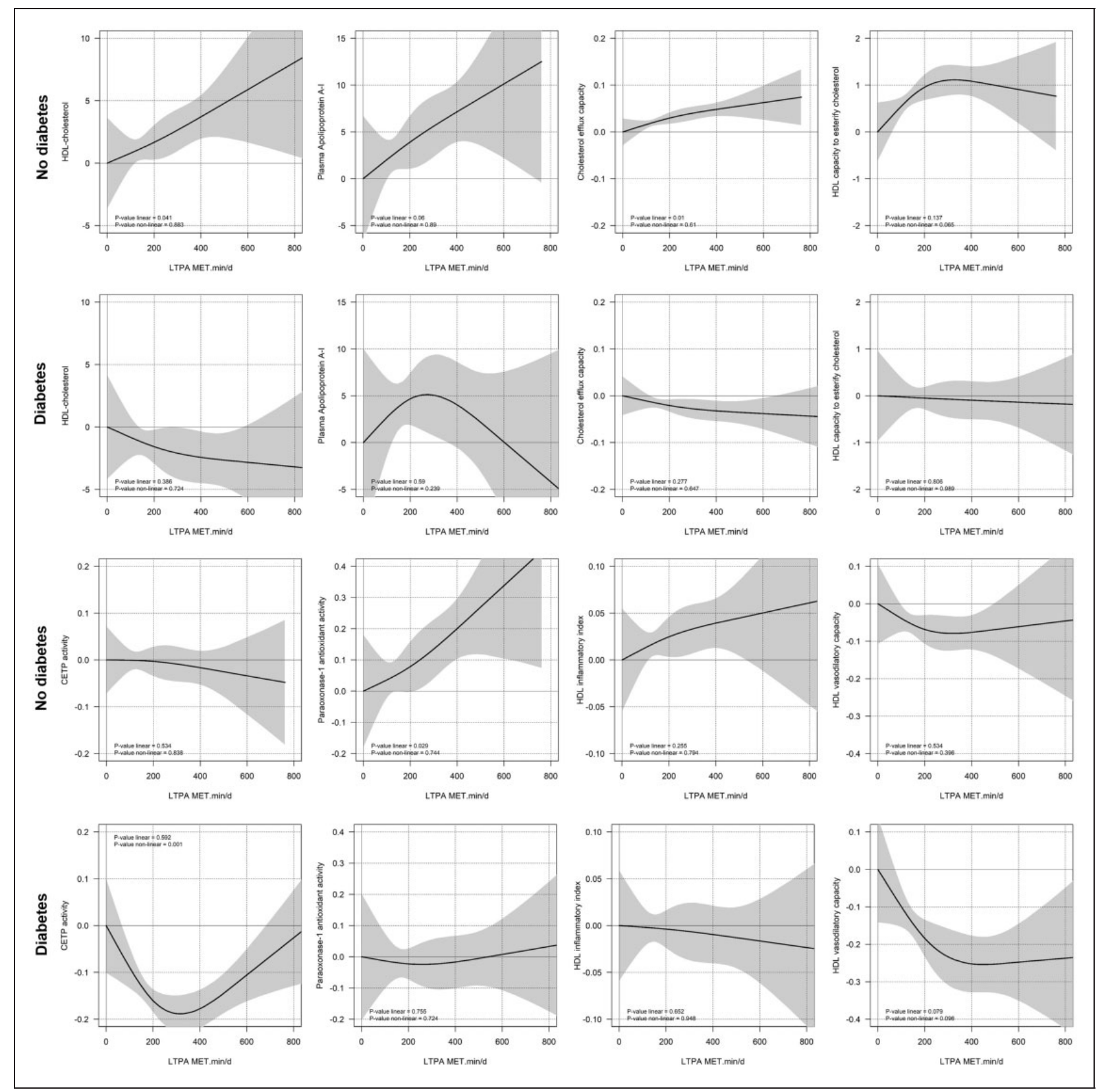

Figure 4. Dose-response association $\dagger$ of HDL-related functional traits assessed by restricted cubic splines, by diabetes status. †Adjusted for sex, intervention group, study centre and baseline age, body mass index, smoking status, Mediterranean diet score, dyslipidemia and hypertension status.

nature of nitric oxide in cardiovascular health, further research regarding the association between long-term higher levels of physical activity and HDL vasodilatory capacity values is necessary.

Taking physical inactivity (0 METs.min/day in LTPA) as the reference, most associations were observed from 100 METs.min/day (700 METs.min/ week), which corresponds to 25 minutes of brisk walking per day, and is just above the World Health Organization guidelines recommendation. ${ }^{23}$ This is consistent with results from a recent meta-analysis of prospective studies that showed a decrease in the risk of a wide range of chronic diseases, including cardiovascular disease and cancer from levels of 86 METs.min/ day (600 METs.min/week). ${ }^{38}$ Moreover, our data suggest no additional benefits beyond 300 METs.min/day (2100 METs.min/week), which is consistent with analyses linking physical activity with the risk of ischaemic heart disease and stroke that show that major gains were achieved at lower levels of physical activity, 
whereas the decrease in risk flattens and becomes minimal at levels higher than 3000-4000 METs.min/ week. ${ }^{38}$

\section{Strengths and limitations}

Our study has several strengths, including the novelty of investigating physical activity in an observational setting in relation to HDL functionality, the prospective cumulative average assessment of physical activity and HDL functionality to increase precision, the comprehensive assessment of an array of HDL-related functional traits using standardised protocols and the relatively large sample size compared to studies in the HDL functionality literature. However, several limitations should be considered when interpreting the present findings. First, the population under study consisted of older adults at high risk of cardiovascular disease, who present characteristics, including more dysfunctional HDL, that cannot be generalisable to healthier populations. Second, this study was a dietary intervention trial which did not include any physical activity advice. As a consequence, we analysed it as a cohort study, and tried to overcome this limitation by adjusting for the intervention group and by taking as the exposure the cumulative average of physical activity from baseline and at one year, which takes into account any change in physical activity during the first year of the trial. Third, the levels of physical activity were self-reported, and not objectively measured, therefore prone to measurement error. Fourth, we used cellular models to determine two HDL functions (CEC and vasodilatory capacity), which may not reflect the biological interplay and potential counterregulatory mechanisms occurring. Fifth, the capacity of HDL to esterify cholesterol determination was performed on HDL isolated by ultracentrifugation, whereas the other assays were performed on apolipoprotein-B-depleted plasma, which might have yielded a different amount of isolated HDLs. Finally, although the total sample size was nearly 300 participants, there were substantial missing data on three assays, namely $23 \%$ for CETP and PON1 activity and $17 \%$ for vasodilatory capacity. However, it is reasonable to assume that these data were missing completely at random, and do not affect the representativeness of the subsample, only larger standard errors and loss of statistical power.

To conclude, we present the first study to assess the long-term associations of real-life physical activity levels and a comprehensive set of HDL functionality properties in high cardiovascular risk older individuals. We found that not only is LTPA associated with greater levels of HDL-cholesterol and ApoA-I, but also with more functional HDL, with greater CEC, esterification capacity, PON1 activity in individuals free of diabetes, and lower CETP activity at intermediate physical activity levels in type 2 diabetes patients. Considering the functionality of HDL rather than its cholesterol content is increasingly gaining interest as a predictor of the risk of cardiovascular disease, therefore more longitudinal studies are needed to evaluate how physical activity influences HDL-related functional traits.

\section{Acknowledgements}

The author(s) are grateful to Daniel Muñoz-Aguayo, Gemma Blanchart and Sonia Gaixas for their technical assistance.

\section{Author contribution}

CL designed and conducted the statistical analysis. AH and MFitó designed the study and $\mathrm{AH}$ acquired the data. MAMG, ER, XP, RE, JSS, DC, AAG, LSM, MFiol, JL, EGG and MFitó contributed with biological samples and/ or participated in the design and development of the clinical trial. CL and AH drafted the manuscript, which was critically revised by MTSF, OC, HS, MAMG, ER, XP, RE, JSS, DC, AAG, LSM, MFiol, JL, EGG and MFitó.

\section{Declaration of conflicting interests}

The author(s) declared the following potential conflicts of interest with respect to the research, authorship, and/or publication of this article: XP reports personal fees from Abbott, Esteve, Lacer, Rubio and Sanofi outside the submitted work. RE reports grants from Bicentury SA, Cerveza y Salud, Grand Fountaine and Novartis SA; and personal fees from Brewers of Europe, FIVIN, Fundación Cerveza y Salud, Lilly Laboratories and Wine and Culinary International Forum outside the submitted work. JSS reports to be a board member and grants from Nut and Dried Fruit; personal fees from Aguas Font Vella Lanjarón, Danone SA and Instituto Danone; grants from Eroski Distributors; and non-financial support from Nuts for Life outside the submitted work. ER reports personal fees and non-financial support from Merck, Sharp \& Dohme; grants, personal fees and nonfinancial support from California Walnut Commission; grants and personal fees from Sanofi; personal fees and non-financial support from Ferrer International; and grants from Pfizer outside the submitted work. The rest of the authors have no conflicts of interest to declare.

\section{Funding}

The author(s) disclosed receipt of the following financial support for the research, authorship, and/or publication of this article: This work was supported by the Beatriu de Pinós postdoctoral programme of the Government of Catalonia's Secretariat for Universities and Research of the Ministry of Economy and Knowledge (2017-BP-00021), Agència de Gestió d'Ajuts Universitaris i de Recerca AGAUR (2014SGR-240, 2017-SGR-222, 2015-FI_B-01042) and the 
Instituto de Salud Carlos III (CB06/03/0028, CD17/00122, CES12/025, JR14/00008, PI11/01647 and PI15/00047). The CIBEROBN and CIBERESP are an initiative of the Instituto de Salud Carlos III, which is supported by FEDER funds.

\section{References}

1. Piepoli MF, Hoes AW, Agewall S, et al. 2016 European Guidelines on cardiovascular disease prevention in clinical practice: the Sixth Joint Task Force of the European Society of Cardiology and Other Societies on Cardiovascular Disease Prevention in Clinical Practice (constituted by representatives of 10 societies and by invited experts). Developed with the special contribution of the European Association for Cardiovascular Prevention \& Rehabilitation (EACPR). Eur Heart $J$ 2016; 37: 2315-2381.

2. Gordon T, Castelli WP, Hjortland MC, et al. High density lipoprotein as a protective factor against coronary heart disease. The Framingham Study. Am J Med 1977; 62: 707-714.

3. Holmes MV, Asselbergs FW, Palmer TM, et al. Mendelian randomization of blood lipids for coronary heart disease. Eur Heart $J$ 2015; 36: 539-550.

4. Voight BF, Peloso GM, Orho-Melander M, et al. Plasma HDL cholesterol and risk of myocardial infarction: a mendelian randomisation study. Lancet 2012; 380: 572-580.

5. Allara E, Morani G, Carter P, et al. Genetic determinants of lipids and cardiovascular disease outcomes: a wideangled mendelian randomization investigation. Circ Genom Precis Med 2019; 12: e002711.

6. Boekholdt SM, Arsenault BJ, Hovingh GK, et al. Levels and changes of HDL cholesterol and apolipoprotein A-I in relation to risk of cardiovascular events among statintreated patients: a meta-analysis. Circulation 2013; 128 : 1504-1512.

7. Keene D, Price C, Shun-Shin MJ, et al. Effect on cardiovascular risk of high density lipoprotein targeted drug treatments niacin, fibrates, and CETP inhibitors: metaanalysis of randomised controlled trials including 117,411 patients. BMJ 2014; 349: g4379.

8. Rosenson RS, Brewer HB Jr, Ansell B, et al. Translation of high-density lipoprotein function into clinical practice: current prospects and future challenges. Circulation 2013; 128: $1256-1267$.

9. Kodama S, Tanaka S, Saito K, et al. Effect of aerobic exercise training on serum levels of high-density lipoprotein cholesterol: a meta-analysis. Arch Intern Med 2007; 167: 999-1008.

10. Ruiz-Ramie JJ, Barber JL and Sarzynski MA. Effects of exercise on HDL functionality. Curr Opin Lipidol 2019; 30: 16-23.

11. Khan AA, Mundra PA, Straznicky NE, et al. Weight loss and exercise alter the high-density lipoprotein lipidome and improve high-density lipoprotein functionality in metabolic syndrome. Arterioscler Thromb Vasc Biol 2018; 38: 438-447.
12. Sarzynski MA, Ruiz-Ramie JJ, Barber JL, et al. Effects of increasing exercise intensity and dose on multiple measures of HDL (high-density lipoprotein) function. Arterioscler Thromb Vasc Biol 2018; 38: 943-952.

13. Pagonas N, Vlatsas S, Bauer F, et al. The impact of aerobic and isometric exercise on different measures of dysfunctional high-density lipoprotein in patients with hypertension. Eur J Prev Cardiol 2019; 26: 1301-1309.

14. Estruch R, Ros E, Salas-Salvado J, et al. Primary prevention of cardiovascular disease with a Mediterranean diet supplemented with extra-virgin olive oil or nuts. N Engl J Med 2018; 378: e34.

15. Martinez-Gonzalez MA, Corella D, Salas-Salvado J, et al. Cohort profile: design and methods of the PREDIMED study. Int J Epidemiol 2012; 41: 377-385.

16. Hernaez A, Castaner O, Elosua R, et al. Mediterranean diet improves high-density lipoprotein function in highcardiovascular-risk individuals: a randomized controlled trial. Circulation 2017; 135: 633-643.

17. Hernaez A, Sanllorente A, Castaner O, et al. Increased consumption of virgin olive oil, nuts, legumes, whole grains, and fish promotes HDL functions in humans. Mol Nutr Food Res 2019; 63: e1800847.

18. Clarke R, Shipley M, Lewington S, et al. Underestimation of risk associations due to regression dilution in long-term follow-up of prospective studies. Am J Epidemiol 1999; 150: 341-353.

19. Elosua R, Garcia M, Aguilar A, et al. Validation of the Minnesota leisure time physical activity questionnaire in Spanish women. Investigators of the MARATDON Group. Med Sci Sports Exerc 2000; 32: 1431-1437.

20. Elosua R, Marrugat J, Molina L, et al. Validation of the Minnesota leisure time physical activity questionnaire in Spanish men. The MARATHOM Investigators. Am J Epidemiol 1994; 139: 1197-1209.

21. Schroder H, Fito M, Estruch R, et al. A short screener is valid for assessing Mediterranean diet adherence among older Spanish men and women. J Nutr 2011; 141: 1140-1145.

22. Wong NKP, Nicholls SJ, Tan JTM, et al. The role of high-density lipoproteins in diabetes and its vascular complications. Int J Mol Sci 2018; 19: 1680.

23. World Health Organization. Global Physical Activity Questionnaire (GPAQ) Analysis Guide. Secondary Global Physical Activity Questionnaire (GPAQ) Analysis Guide. https://www.who.int/ncds/surveillance/ steps/resources/GPAQ_Analysis_Guide.pdf. (accessed 2 May 2020).

24. Ostman C, Smart NA, Morcos D, et al. The effect of exercise training on clinical outcomes in patients with the metabolic syndrome: a systematic review and metaanalysis. Cardiovasc Diabetol 2017; 16: 110.

25. Richter EA and Ruderman NB. AMPK and the biochemistry of exercise: implications for human health and disease. Biochem $J$ 2009; 418: 261-275.

26. Garcia D and Shaw RJ. AMPK: mechanisms of cellular energy sensing and restoration of metabolic balance. $\mathrm{Mol}$ Cell 2017; 66: 789-800. 
27. Herzig S and Shaw RJ. AMPK: guardian of metabolism and mitochondrial homeostasis. Nat Rev Mol Cell Biol 2018; 19: 121-135.

28. Duval C, Muller M and Kersten S. PPARalpha and dyslipidemia. Biochim Biophys Acta 2007; 1771: 961-971.

29. Greer EL, Banko MR and Brunet A. AMP-activated protein kinase and FoxO transcription factors in dietary restriction-induced longevity. Ann NY Acad Sci 2009; 1170: 688-692.

30. Klotz LO, Sanchez-Ramos C, Prieto-Arroyo I, et al. Redox regulation of FoxO transcription factors. Redox Biol 2015; 6: 51-72.

31. Mo C, Wang L, Zhang J, et al. The crosstalk between Nrf2 and AMPK signal pathways is important for the anti-inflammatory effect of berberine in LPS-stimulated macrophages and endotoxin-shocked mice. Antioxid Redox Signal 2014; 20: 574-588.

32. Aviram M and Vaya J. Paraoxonase 1 activities, regulation, and interactions with atherosclerotic lesion. Curr Opin Lipidol 2013; 24: 339-344.

33. Kontush A and Chapman MJ. Functionally defective high-density lipoprotein: a new therapeutic target at the crossroads of dyslipidemia, inflammation, and atherosclerosis. Pharmacol Rev 2006; 58: 342-374.

34. Wang P, Tait SM and Chaudry IH. Sustained elevation of norepinephrine depresses hepatocellular function. Biochim Biophys Acta 2000; 1535: 36-44.

35. Kemps H, Krankel N, Dorr M, et al. Exercise training for patients with type 2 diabetes and cardiovascular disease: what to pursue and how to do it. A Position Paper of the European Association of Preventive Cardiology (EAPC). Eur J Prev Cardiol 2019; 26: 709-727.

36. Barter P and Rye KA. Cholesteryl ester transfer protein: its role in plasma lipid transport. Clin Exp Pharmacol Physiol 1994; 21: 663-672.

37. Nosarev AV, Smagliy LV, Anfinogenova Y, et al. Exercise and NO production: relevance and implications in the cardiopulmonary system. Front Cell Dev Biol 2014; 2: 73.

38. Kyu HH, Bachman VF, Alexander LT, et al. Physical activity and risk of breast cancer, colon cancer, diabetes, ischemic heart disease, and ischemic stroke events: systematic review and dose-response meta-analysis for the Global Burden of Disease Study 2013. BMJ 2016; 354: i3857. 